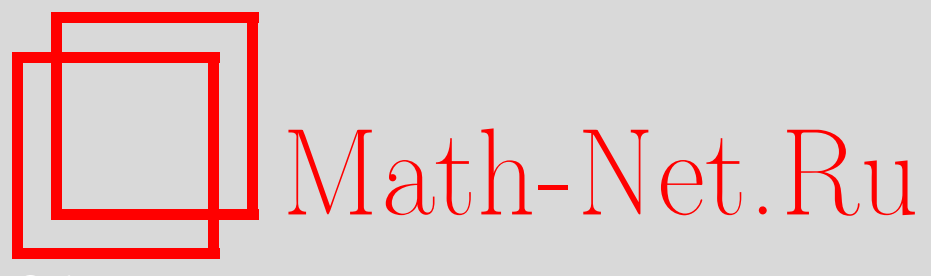

А. И. Алексенко, А. Ю. Плахов, Об аэродинамической задаче Ньютона для невыпуклых тел, УМН, 2008, том 63, выпуск 5, 183-184

DOI: https://doi.org/10.4213/rm9189

Использование Общероссийского математического портала Math-Net.Ru подразумевает, что вы прочитали и согласны с пользовательским соглашением http://www . mathnet.ru/rus/agreement

Параметры загрузки:

IP : 54.198 .64 .247

26 апреля 2023 г., 10:11:54

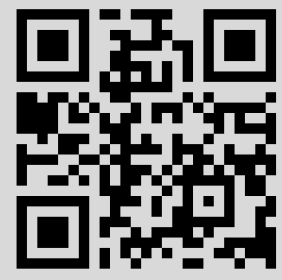




\section{Об аэродинамической задаче Ньютона для невыпуклых тел}

\section{А. И. Алексенко, А. Ю. Плахов}

Задача о теле наименьшего сопротивления заключается в следующем. Рассматривается евклидово пространство $\mathbb{R}^{3}$ с координатами $x, y, z$. На тело - связное компактное множество с кусочно гладкой границей - падает однородный поток точечных частиц со скоростью $(0,0,-1)$. Частицы, налетающие на тело, совершают одно или несколько упругих отражений от его поверхности и затем движутся свободно. Между собой частицы не взаимодействуют. Каждая частица передает телу некоторый импульс; тем самым создается сила давления на тело, которую принято называть (аэродинамическим) сопротивлением. Задача заключается в том, чтобы из некоторого класса допустимых тел выбрать тело с наименьшим сопротивлением.

Впервые эта задача была рассмотрена Ньютоном в 1686 г. в классе выпуклых и осесимметричных тел, вписанных в фиксированный прямой круговой цилиндр. Ось симметрии тела и ось цилиндра совпадают с координатной осью Oz. Решение представляет собой тело вращения, ограниченное сверху и снизу плоскими дисками. В начале 1990-х годов вновь возник интерес к этой задаче. В работах Буттаццо, Каволя, Лашан-Робера и других рассмотрен более широкий класс тел, не обладающих свойством симметрии (т. е. вписанных в заданный цилиндр и выпуклых). Оказалось, что решение в этом классе не совпадает с решением Ньютона (см. работы [1]-[5]).

Если опустить оба свойства симметрии и выпуклости, то получится класс произвольных тел, вписанных в цилиндр. Предполагается, что тело из данного класса содержится в цилиндре и содержит одно из перпендикулярных круговых сечений цилиндра. Доказано, что нижняя грань сопротивления в этом классе равна нулю [6].

Цель настоящей работы - рассмотреть четвертый логически возможный случай: класс тел, обладающих осевой симметрией, но (вообще говоря) невыпуклых. На плоскости $\mathbb{R}^{2}$ с координатами $x, z$ рассмотрим прямоугольник $[0,1] \times[0, h]$ и множество $F$, вписанное в этот прямоугольник. Другими словами, $F$ принадлежит прямоугольнику и содержит точки каждой их его четырех сторон. Кроме того, $F$ замкнуто, связно, симметрично относительно оси $O z$, имеет кусочно гладкую границу и обладает свойством регулярности биллиардного рассеяния в $\mathbb{R}^{2} \backslash F$. Это свойство заключается в том, что для почти всех $x \in[0,1]$ движение биллиардной частицы, имеющей координаты $x(t)=x, z(t)=-t$ при $t \leqslant-h$, определено при всех $t \in \mathbb{R}$. Частица совершает конечное число отражений от границы $\partial F$, а затем движется свободно с некоторой скоростью $v_{F}(x) \in S^{1}$. В свойство регулярности мы включаем также условие о том, что $v_{F}(x)=\left(v_{F}^{x}(x), v_{F}^{z}(x)\right)$ есть измеримая функция от $x$. Обозначим $\mathscr{F}_{h}$ класс всех таких множеств $F$. Сопротивление тела $\Omega_{F}$, полученного вращением множества $F \in \mathscr{F}_{h}$ вокруг оси $O z$, равно $-2 \pi \rho(0,0, R(F))$, где $\rho$ - плотность потока, а $R(F)=\int_{0}^{1}\left(1+v_{F}^{z}(x)\right) x d x$. Наша задача - минимизировать сопротивление в классе тел вращения $\Omega_{F}, F \in \mathscr{F}_{h}$; другими словами, найти $\inf _{F \in \mathscr{F}_{h}} R(F)$.

Обозначим $\mathscr{F}_{h}^{\text {conv }}$ класс выпуклых множеств $F \in \mathscr{F}_{h}$. Верхняя часть границы каждого множества $F \in \mathscr{F}_{h}^{\text {conv }}$ есть график некоторой функции $z=h-f_{F}(|x|)$, где $f_{F}(0)=0, f_{F}(1) \leqslant h$, функция $f_{F}$ выпукла на $[0,1]$ и монотонно не убывает. Определим модифицированный закон отражения от границы множества $F \in \mathscr{F}_{h}^{\text {conv }}$ следующим образом. Частица первоначально движется согласно $x(t)=x \in[-1,1]$, $z(t)=-t$, а после отражения приобретает скорость $\hat{v}_{F}(x)$, параллельную касательной к $\partial F$ в точке отражения: $\hat{v}_{F}(x)=\left(\hat{v}_{F}^{x}(x), \hat{v}_{F}^{z}(x)\right)=\left(\operatorname{sgn} x,-f_{F}^{\prime}(|x|)\right) / \sqrt{1+f_{F}^{\prime 2}(|x|)}$.

Работа выполнена при поддержке Centre for Research on Optimization and Control $(C E O C)$ из "Fundaçao para a Ciência e a Tecnologia" (FCT) и European Community Fund FEDER/POCTI, а также фонда FCT PTDC/MAT/72840/2006. 
Соответствующий функционал сопротивления имеет вид $\widehat{R}(F)=\int_{0}^{1}\left(1+\hat{v}_{F}^{z}(x)\right) x d x$. Таким образом, $\widehat{R}(F)$ есть сопротивление выпуклого осесимметричного тела $\Omega_{F}$ для процесса рассеяния, при котором отраженная частица движется по касательной.

Следующая теорема позволяет свести задачу о минимизации сопротивления $R$ в классе тел вращения к задаче о минимизации $\widehat{R}$ в классе выпуклых тел вращения.

TeOpema 1. $\inf _{F \in \mathscr{F}_{h}} R(F)=\inf _{F \in \mathscr{F}_{h}^{\text {conv }}} \widehat{R}(F)$.

Эта теорема вытекает из двух лемм, доказательство которых мы опускаем.

Лемма 1. Пусть $F \in \mathscr{F}_{h}$. (Заметим, что выпуклая оболочка conv $F$ принадлежит $\left.F_{h}^{\text {conv }}\right)$. Справедливо неравенство $\widehat{R}(\operatorname{conv} F) \leqslant R(F)$.

Лемма 2. Пусть $F \in \mathscr{F}_{h}^{\text {conv }}$. Тогда существует последовательность невыпуклых множеств $F_{n} \in \mathscr{F}_{h}$ таких, ито $\lim _{n \rightarrow \infty} R\left(F_{n}\right)=\widehat{R}(F)$.

Поиск минимума $\widehat{R}$ сводится к минимизации функционала $\int_{0}^{1}\left(1-\frac{f^{\prime}(x)}{\sqrt{1+f^{\prime 2}(x)}}\right) x d x$ в классе выпуклых и неубывающих на $[0,1]$ функций $f$, удовлетворяющих соотношениям $f(0)=0, f(1) \leqslant h$. Эта минимизация проводится с помощью принципа максимума Понтрягина; в результате получаем следующую теорему.

Теорема 2. Выполнено равенство

$$
\inf _{F \in \mathscr{F}_{h}^{\text {conv }}} \widehat{R}(F)=\frac{1}{2}-\frac{1}{16}\left(8-2 c^{2 / 3}-3 c^{4 / 3}\right) \sqrt{1-c^{2 / 3}}+\frac{3 c^{2}}{16} \ln \left(\frac{1+\sqrt{1-c^{2 / 3}}}{c^{1 / 3}}\right),
$$

где величина с однозначно определяется по параметру $h$ :

$$
h=\int_{c}^{1} \sqrt{\left(\frac{x}{c}\right)^{2 / 3}-1} d x=-\frac{3}{8}\left(\left(c^{1 / 3}-2 c^{-1 / 3}\right) \sqrt{1-c^{2 / 3}}-\ln \left(\frac{1+\sqrt{1-c^{2 / 3}}}{c^{1 / 3}}\right)\right) .
$$

Множество $\widehat{F}_{h} \in \mathscr{F}_{h}^{\text {conv }}$, минимизирующее функционал $\widehat{R}$, задается соотношениями $0 \leqslant z \leqslant h$, если $|x| \leqslant c$, и $0 \leqslant z \leqslant h-\int_{c}^{x} \sqrt{(t / c)^{2 / 3}-1} d t$, если $c<|x| \leqslant 1$. Не существует множества, на котором достигается минимум функционала $R$; однако для всякой минимизирующей последовательности множеств $F_{h}^{n} \in \mathscr{F}_{h}$ граница выпуклой оболочки $\partial\left(\operatorname{conv} F_{h}^{n}\right)$ при $n \rightarrow \infty$ аппроксимирует границу $\partial \widehat{F}_{h}$ в классе $C^{1}$.

Обозначим $\mathscr{R}(h):=\inf _{F \in \mathscr{F}_{h}} R(F)$ и $\mathscr{R}_{N}(h):=\inf _{F \in \mathscr{F}_{h}^{\text {conv }}} R(F)$ наименьшее значение сопротивления для нашей задачи и задачи Ньютона соответственно. Справедливы следующие асимптотические соотношения: $\mathscr{R}\left(0^{+}\right)=(1 / 2) \mathscr{R}_{N}\left(0^{+}\right)=1 / 2$; $\mathscr{R}(h)=(1 / 4) \mathscr{R}_{N}(h)(1+o(1))=(27 / 128)(1+o(1)) / h^{2}$ при $h \rightarrow+\infty$.

\section{Список литературы}

[1] G. Buttazzo, B. Kawohl, Math. Intelligencer, 15:4 (1993), 7-12. [2] F. Brock, V. Ferone, B. Kawohl, Calc. Var. Partial Differential Equations, 4:6 (1996), 593-599. [3] T. Lachand-Robert, M. A. Peletier, Math. Nachr., 226:1 (2001), 153-176. [4] T. Lachand-Robert, E. Oudet, SIAM J. Optim., 16:2 (2005), 368-379. [5] D. Bucur, G. Buttazzo, Variational methods in shape optimization problems, Birkhäuser, Boston, MA, 2005. [6] А. Ю. Плахов, УМН, 58:1 (2003), 195-196.

А. И. Алексенко (А. I. Aleksenko)

Университет Авейро, Португалия

E-mail: alena-aleksenko@rambler.ru
Представлено В. М. Тихомировым Принято редколлегией 17.03.2008

А. Ю. Плахов (А. Yu. Plakhov)

Университет Аберстуит, Великобритания

E-mail: axp@aber.ac.uk 